QCD Evolution Workshop 2014

International Journal of Modern Physics: Conference Series

Vol. 37 (2015) 1560038 (9 pages)

(C) The Authors

DOI: $10.1142 / \mathrm{S} 2010194515600381$

\title{
Angular Momentum and Polarization in Hadron Collisions up to LHC Energies
}

\author{
Gary R. Goldstein \\ Department of Physics and Astronomy, Tufts University, \\ Medford, MA 02155, USA \\ gary.goldstein@tufts.edu \\ Simonetta Liuti \\ Physics Department, University of Virginia 382 McCormick Rd., \\ Charlottesville, Virginia 22904, USA \\ sl4y@virginia.edu
}

Published 25 February 2015

\begin{abstract}
Longstanding puzzles in spin physics can be confronted at the high energies of the LHC. Heavy quarks will be produced with significant polarization, both as single spin asymmetries and through polarization correlations. Lower energy proton accelerator and leptoproduction data suggest various mechanisms within QCD for polarization phenomena that can be tested at higher energies. Observation of strange and charm hadron polarization reveals important aspects of QCD spin physics. Top quark polarization is predicted to be significant, and polarization correlations will reveal important aspects of the gluon distributions of the hadrons.
\end{abstract}

\section{Large Polarization in Hadron Processes}

The inclusive production of highly polarized strange hyperons is a long-standing puzzle in hadronic spin physics. The expectation from Perturbative QCD based on the Kane, Pumplin, Repko (KPR) calculation [1] was that such polarization should be small as governed by the quantity $\alpha_{s}(\hat{s}) m_{q} / \sqrt{\hat{s}}$, where $m_{q}$ is the effective mass of the strange quark (or the hyperon) that normalizes the helicity flip vertices, and $\sqrt{\hat{s}}$ a characteristic energy of the hard production process for the quark. Explanations for why measurements showing an increasing negative polarization, up to $p_{T} \approx 2 \mathrm{GeV}$, did not agree with the expectation have been proposed over the years. Such striking behavior has been explored and awaits confirmation from higher energy collisions at the LHC [2]. Among the various mechanisms that were proposed,

This is an Open Access article published by World Scientific Publishing Company. It is distributed under the terms of the Creative Commons Attribution 3.0 (CC-BY) License. Further distribution of this work is permitted, provided the original work is properly cited. 
Dharmaratna and Goldstein [3] calculated all the Next to Leading Order (NLO) hard QCD amplitudes that enter in hadron+hadron collisions via parton+parton producing s-quark pairs or heavier flavors. They found that polarizations at the several percent level, increasing with quark or hyperon mass, were produced through interference between these amplitudes and tree level terms. The kinematic dependence of these results favored small $p_{T}$. However, if this hard process is the source of measured hyperon polarization, that polarization must be enhanced through soft process hadronization. This was accomplished in [3] through an ad hoc simple ansatz for that soft process by analogy with the "Thomas precession" mechanism [4] to boost the $x_{F}$ (quark) to $x_{F}$ (hadron) while enhancing the scale of the polarization. Although this scheme could explain the systematic behavior of $\Lambda$ and $\Sigma$ polarization, including [5] the behavior of the $\Lambda_{c}$ data from Fermilab experiment E791 [6], the need to introduce a soft mechanism beyond the NLO PQCD treatment suggests a more rigorous QCD based formulation [7]. This will be elucidated below. First we consider the electroproduction of hyperons.

\section{Leptoproduction of Hyperons}

Several directions have been considered to explore hyperon and heavy flavor baryon polarization phenomena as applied to LHC energies, where the $g+g$ mechanism is dominant, especially at small Bjorken $x$ for both protons. In parallel with the purely hadronic processes, the leptoproduction framework is studied. In all these cases of single spin asymmetries, transversity is a crucial ingredient.

To quickly review, transversity is the spin quantization of a moving particle in a direction normal to the direction of motion [8]. Since it involves moving particles, its definition is through a Pauli-Lubanski tensor, or, more transparently, a linear combination of helicities. For spin $1 / 2$ the transversity, $\tilde{\lambda}$, is

$$
\mid p, \tilde{\lambda}= \pm)=\frac{1}{2}(|p, \lambda=+\rangle \pm i|p, \lambda=-\rangle)
$$

This corresponds to a $\mathrm{Y}$-axis spin quantization for a particle at rest, but now moving in the X-Z plane. Quantizing in the X-direction would entail replacing the "i" by 1. For Dirac particles the transversity is not "transverse spin", since the upper and lower Dirac spinor components transform differently for helicity \pm . (But the combinations of helicities that correspond to transversity remain unchanged under boosts along the particle's 3-momentum direction.) In TMDs or GPDs for which the transversity of the nucleon or the quark is fixed, the associated distribution must involve helicity flip. Hence for the "observation" of a single quark or nucleon with definite transversity there has to be a chiral odd function. The pdf $h_{1}(x)$ is a measure of the transfer of transversity from the nucleon to the quark - it is a double polarization correlation. Its first moment is the tensor charge, $\delta q$ for quark flavor $q$.

Transversity can be accessed experimentally through SIDIS, with a polarized nucleon target and pion production with transverse momentum. The appropriate 


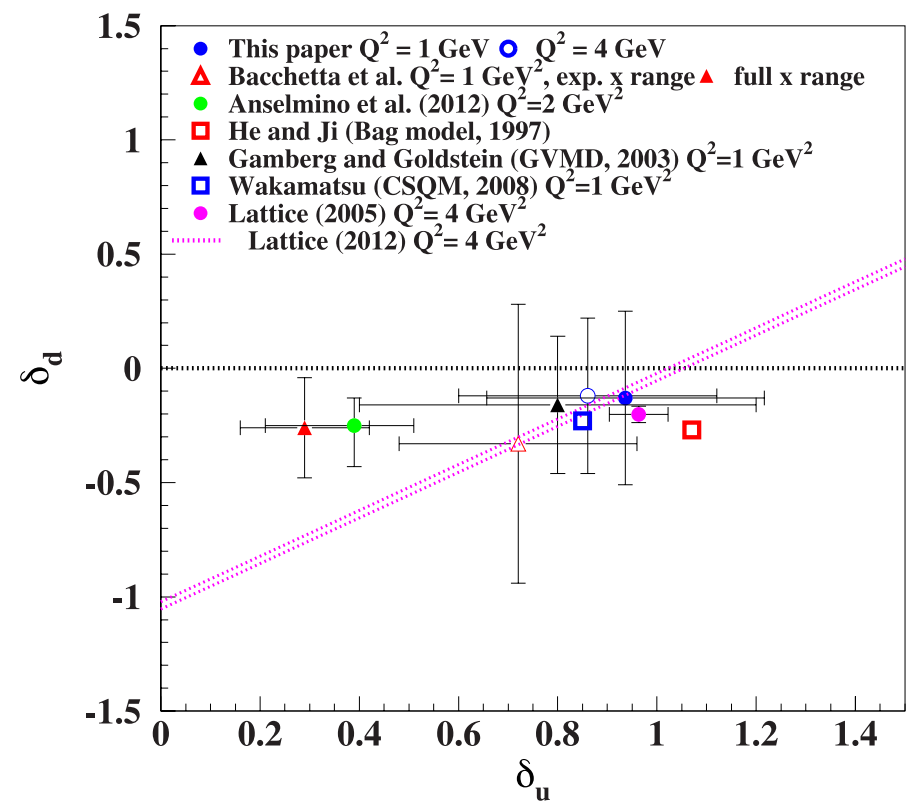

Fig. 1. Predictions and determinations of tensor charges $\delta_{u}, \delta_{d}$, adapted from Ref.[13].

kinematics for the data isolates the convolution of $h_{1}(x)$ and the polarized quark fragmentation function $H_{1}^{\perp}(z)$ [9].

Another, quite independent method, is through the exclusive electroproduction of $\pi^{0}$ or $\eta$. The chiral odd GPDs are involved in this process [10], particularly $H_{T}(x, \xi, t)$, for which $H_{T}(x, 0,0)=h_{1}(x)$. In Ref.[11] we calculated the Chiral Odd GPDs in a flexible Reggeized spectator model and we estimated the values of the tensor charge for $u$ and $d$ quarks using constraints on the model's parameters from the chiral even sector. Measurements of exclusive $\pi^{0}$ electroproduction by Hall B at JLab [12] will allow us to determine directly the transversity distribution and the tensor charge for up and down quarks. In Ref. [13] (see also Figure 1) we show a compilation of both extractions of the tensor charge from data and model calculations (see also Ref.[14] for another approach including chiral odd GPDs in the phenomenology).

\section{Generalized Fracture Functions}

A novel approach where transverse polarization can be generated is through the QCD based description of target fragmentation within the "fracture function" picture $[15,16]$. This can be a leading twist result in which a soft gluon exchange provides the necessary phase, provided the $\Lambda$ has non-zero $p_{T}$ relative to the $\gamma^{*}$ direction. This is possible, whether a current fragment is observed or not, because there is a hadronic plane established. 
The fracture functions are joint probabilities that relate the beam direction hard scattered quark distribution from the target nucleon (a function of $x_{B j}$ ) to the baryon fragmentation of the target (a function of $z$ ). For a single spin asymmetry (SSA) there must be an interference, which requires some process like a final state interaction. Formally, for a quark-nucleon FF (see Ref.[17] for a detailed treatment),

$$
\begin{aligned}
\mathcal{F}_{\Lambda_{N} ; \Lambda_{\Lambda}^{\prime}, \Lambda_{\Lambda}}^{\lambda_{q}} & \left(x, k_{T}, z, p_{T}, Q^{2}\right) \\
= & \sum_{\Lambda_{X}} \int \frac{d^{3} P_{X}}{(2 \pi)^{3} 2 E_{X}} \int \frac{d^{4} \xi}{(2 \pi)^{4}} e^{i k \cdot \xi}\left\langle P, \Lambda_{N}\left|\bar{\psi}^{\lambda_{q}}(\xi)\right| P_{h}, \Lambda_{\Lambda}^{\prime} ; X\right\rangle \\
& \times\left\langle P_{h}, \Lambda_{\Lambda} ; X\left|\psi^{\lambda_{q}}(0)\right| P, \Lambda_{N}\right\rangle,
\end{aligned}
$$

where transverse momenta $k_{T}, p_{T}$ are for the quark and the outgoing hadron, respectively. Since we are interested in the unpolarized cross section or the transverse $\Lambda$ polarization, we keep the definition here in terms of diagonal helicities for the quark fields and the target nucleon, while allowing for change of helicity for the outgoing hadron. The behavior of FFs [18] for $\Lambda$ electroproduction can be evaluated within the spectator model (see also [19]). This model successfully described a large array of phenomena. We considered a FF at the tree level for the process $\gamma^{*}+N \rightarrow \bar{s}+\Lambda+X$ where the $\bar{s}$-quark hadronizes and $X$ contains unmeasured, inclusive hadrons.

In the spectator model for the nucleon to a light quark transition, the spectator is the (ud) or ( $\mathrm{uu}$ ) diquark - scalar or axial vector. We take a scalar diquark to begin with. Then the struck u-quark (with $x$ and $k_{T}$ ) leaves the diquark (with $(1-x)$ and $-k_{T}$ ) to fragment into the $\Lambda$ (with $z$ and $p_{T}$ ). The intermediate state is a single diquark, either scalar or axial vector. In this spectator model with the scalar diquark we can write

$$
\mathcal{F}_{\Lambda_{N} ; \Lambda_{\Lambda}^{\prime}, \Lambda_{\Lambda}}^{\lambda_{q}}\left(x, k_{T}, z, p_{T}, Q^{2}\right)=A_{\Lambda_{N}, \lambda_{q}} \sum_{\Lambda_{X}} B_{\Lambda_{X}}^{\Lambda_{\Lambda}, \Lambda_{\Lambda}^{\prime}}
$$

where

$$
A_{\Lambda_{N}, \lambda_{q}}=\left|\phi_{\lambda_{q}, \Lambda_{N}}(k, P)\right|^{2}
$$

and

$$
B_{\Lambda_{X}}^{\Lambda_{\Lambda}, \Lambda_{\Lambda}^{\prime}}=\tilde{\phi}_{\Lambda_{X}, \Lambda_{\Lambda}^{\prime}}^{*}\left(P_{X}, P_{h}\right) \tilde{\phi}_{\Lambda_{X}, \Lambda_{\Lambda}}\left(P_{X}, P_{h}\right)
$$

with each helicity structure given by a scalar coupling defining the helicity structures at each soft vertex.

Gauge links have to be carefully implemented for consistency. The unmeasured states, X, are summed over in the inclusive cross section. To approximate all of those states and in keeping with the spectator approach, we take $\mathrm{X}$ to be a single mass, $M_{X}$. Then there is the condition that $\left(P-k-P_{\Lambda}\right)^{2}=m_{s}^{2}$. Given this condition we have the diquark momentum $(P-k)^{2}$ and the quark momentum $k^{2}$ constrained. The struck quark with momentum $(k+q)$ leads to $\delta\left((k+q)^{2}\right) \approx \frac{x_{B j}}{Q^{2}} \delta\left(x-x_{B j}\right)$. 
The unpolarized fracture function is evaluated in this model by summing over the unobservable helicities and integrating over 4 -momentum $k$. The remaining integrals over $k^{-}, \vec{k}_{T}$ are readily evaluated. The $k^{-}=\frac{k^{2}+\vec{k}_{T}^{2}}{x P^{+}}$is fixed for each $\vec{k}_{T}$, so the only independent integral is over $\vec{k}_{T}$. If the struck quark is not "observed", i.e. its fragmentation is not measured, the $\vec{k}_{T}$ can be set to 0 without loss of generality. for quark flavor $q$ (where helicities have been summed or averaged over are as well). Since this sums over all hadrons, the particular hyperon cross section that we are interested in is one component of the sum. We can estimate the bound on its contribution by taking the strange quark contribution $f_{1}^{s}\left(x, Q^{2}\right)$. For the masses that enter the $\phi$ and $\tilde{\phi}$ in Eqs. (4) \& (5) we use the values set in the parameterization of the Reggeized spectator model of Ref. [20].

The unpolarized differential cross section corresponding to that FF is obtained from the relation between the results of Eq. (4) and Eq. (5), which is related to the hadronic tensor for the electroproduction $W_{\mu \nu}$.

To obtain a SSA for a target fragment $\Lambda$ an additional mechanism must be invoked. An "extended FF" that has both an imaginary part and a helicity flip can be constructed using a final state interaction [18]. The model calculations of transversity-odd TMDs [21, 22, 23] for the Sivers and Boer-Mulders functions, as measured in SIDIS, suggest a mechanism for SSA. The TMD polarization result, which has the form

$$
P_{y}=C_{F} \alpha_{s}\left(\mu^{2}\right) \frac{(x M+m) k_{x}}{\left[(x M+m)^{2}+\vec{k}_{\perp}^{2}\right]} \frac{\Lambda\left(\vec{k}_{\perp}^{2}\right)}{\vec{k}_{\perp}^{2}} \ln \left(\frac{\Lambda\left(\vec{k}_{\perp}^{2}\right)}{\Lambda(0)}\right)
$$

with

$$
\Lambda\left(\vec{k}_{\perp}^{2}\right)=\vec{k}_{\perp}^{2}+x(1-x)\left(-M^{2}+\frac{m^{2}}{x}+\frac{\lambda^{2}}{1-x}\right)
$$

will be modified by the FF kinematics, but retaining the same functional form [18].

\section{Top Quark Polarizations and Spin Correlations}

We see that if quarks are polarized in a collision, whether in leptoproduction or hadronic production, the direct measurement of that polarization is indirect, because of confinement. There is an exception to that rule in top quark production. Top quarks, because of their large mass, decay via the weak interaction so rapidly that their polarization is virtually undisturbed by strong effects and is encoded in their decay products [24]. This allows certain spin correlations among decay products to give unambiguous information about the parent top quark polarization [25], as illustrated for the "dilepton channel" in Fig. 2.

For the LHC the primary production mechanism is gluon fusion. That can also be a source for single polarization of only one member of the $t-\bar{t}$ pair. Extending the gluon fusion mechanism of Ref. [3] to top quark masses and at LHC energies, shows that single top quark spin asymmetry may be a few percent and will be measurable. 

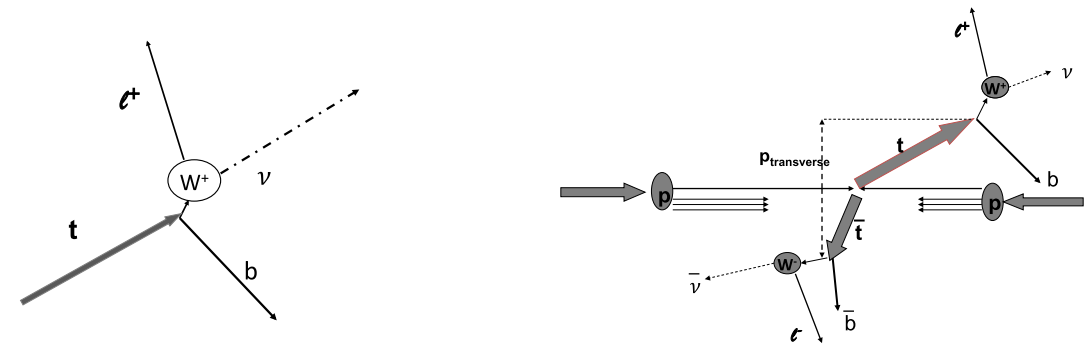

Fig. 2. Leptonic top decay channel (left), dilepton channel (right).

Because gluon fusion is dominant for top pair production, we can ask if polarized gluon distributions will be accessed through top pair spin correlations. For lighter quark pairs the final state interactions before hadronizing could be expected to destroy the coherence of the polarization information. But for the rapid decay for tops, that final state interaction will not be a consideration. The decay products can be well separated spatially, to minimize reinteractions. We have been considering the details for using top pair correlations to specify gluon polarizations.

The QCD amplitudes for $g+g \rightarrow t+\bar{t}$ are well known [3, 26]. With onshell gluons there are 2 helicity values, \pm 1 , leading to 16 hellcity combinations, but parity restricts the number of independent amplitudes to 8 . Let these amplitudes be $A_{\Lambda_{g 1}, \Lambda_{g 2} ; t, \bar{t}}(\hat{s}, \hat{t})$ with $\Lambda_{g 1}, \Lambda_{g 2}$ the gluon helicities, $t, \bar{t}$ the top and antitop helicities, and $\hat{s}, \hat{t}$ the kinematic invariants in an arbitrary frame. Let $g_{\Lambda_{N 1}, \Lambda_{X 1}, \Lambda_{g 1}}^{(1)}\left(x_{1}, k_{T}, M_{X 1}^{2}\right)$ be the amplitude for proton number 1 to emit a gluon no.1 with longitudinal momentum fraction $x_{1}$ and transverse momentum $k_{T}$, along with an unspecified residual $X 1$ of mass $M_{X 1}$, with corresponding $g^{(2)}$ for the other proton. The overall amplitude for $N_{1}+N_{2} \rightarrow t+X_{1}+\bar{t}+X_{2}$ is then $g_{\Lambda_{N 1}, \Lambda_{X 1}, \Lambda_{g 1}}^{(1)} g_{\Lambda_{N 2}, \Lambda_{X 2}, \Lambda_{g 2}}^{(2)} A_{\Lambda_{g 1}, \Lambda_{g 2} ; t, \bar{t}}$. The kinematic variables of the gluon from proton number 1 in $g^{(1)}$ must be matched with the hard amplitude $A$ and the other incoming gluon $g^{(2)}$.

To construct differential cross sections or density matrices for unpolarized colliding protons, this amplitude must be combined with its conjugate and summed and integrated over unobserved quantities including helicities. The terms can be rearranged to correspond to gluon distributions and hard scattering amplitude products,

$$
\sum_{\Lambda_{g 1}, \Lambda_{g 2}, \Lambda_{g 1}^{\prime}, \Lambda_{g 2}^{\prime}}\left(G_{\Lambda_{g 2}^{\prime}, \Lambda_{g 2}}^{(2)} G_{\Lambda_{g 1}^{\prime}, \Lambda_{g 1}}^{(2)}\right) A_{\Lambda_{g 1}^{\prime}, \Lambda_{g 2}^{\prime} ; t^{\prime}, \bar{t}^{\prime}}^{*} A_{\Lambda_{g 1}, \Lambda_{g 2} ; t, \bar{t}}
$$

The two bracketed terms are the (polarized) gluon distributions,

$$
G_{\Lambda_{g 1}, \Lambda_{g 1}^{\prime}}^{(1)}=\sum_{\Lambda_{X 1}, \Lambda_{N 1}} \int_{X_{1}} g_{\Lambda_{N 1}, \Lambda_{X 1}, \Lambda_{g 1}^{\prime}}^{(1) *} g_{\Lambda_{N 1}, \Lambda_{X 1}, \Lambda_{g 1}}^{(1)},
$$


Table 1. Values of double density matrix elements $\rho$ for combinations with linearly polarized (LP) or unpolarized (UP) gluon distributions evaluated in the $t+\bar{t}$ center of mass.

\begin{tabular}{|c|c|c|c|}
\hline$\rho_{t^{\prime}, \bar{t}^{\prime} ; t, \bar{t}}$ & UP,UP & LP,LP & UP,LP + LP,UP \\
\hline,++++ & $\gamma^{-2}\left(1+\beta^{2}\left(1+\sin ^{4} \theta\right)\right)$ & $\gamma^{-2}\left(-1+\beta^{2}\left(1+\sin ^{4} \theta\right)\right)$ & $-4 \gamma^{-2} \beta^{2} \sin ^{2} \theta$ \\
\hline,+-+- & $\left.\beta^{2} \sin ^{2} \theta\left(2-\sin ^{2} \theta\right)\right)$ & $-\beta^{2} \sin ^{4} \theta$ & 0 \\
\hline,++-- & $\gamma^{-2}\left(-1+\beta^{2}\left(1+\sin ^{4} \theta\right)\right)$ & $\gamma^{-2}\left(+1+\beta^{2}\left(1+\sin ^{4} \theta\right)\right)$ & $+4 \gamma^{-2} \beta^{2} \sin ^{2} \theta$ \\
\hline,+--+ & $\beta^{2} \sin ^{4} \theta$ & $\left.-\beta^{2} \sin ^{2} \theta\left(2-\sin ^{2} \theta\right)\right)$ & 0 \\
\hline,+++- & $-2 \gamma^{-1} \beta^{2} \sin ^{3} \theta$ & $-2 \gamma^{-1} \beta^{2} \sin ^{3} \theta$ & $-4 \gamma^{-1} \beta^{2} \sin \theta \cos \theta$ \\
\hline,++-+ & $2 \gamma^{-1} \beta^{2} \sin ^{3} \theta \cos \theta$ & $2 \gamma^{-1} \beta^{2} \sin ^{3} \theta \cos \theta$ & $4 \gamma^{-1} \beta^{2} \sin \theta \cos \theta$ \\
\hline
\end{tabular}

and similarly for $G^{(2)}$.

The gluon distributions can be broken up into definite polarization states, particularly linearly polarized combinations, which are of special interest [7, 27]. Selecting gluons with or without linear polarization (LP or UP) leads to density matrices for different combinations of top helicity states, as shown in Table 1.

The semi-leptonic decays of the top quark afford the best opportunity for polarization analysis [24]. The decay is primarily through the favored $t \rightarrow W^{+}+b$; $W^{+} \rightarrow l^{+}+\nu_{l}$. As shown in Ref. [24], the amplitude $B_{\lambda_{b}, t}$ for a polarized top quark at rest to decay into a measured b-quark and antilepton along with an unobserved neutrino has the simple angular dependence $\propto\left(I+\vec{p}_{\bar{l}} \cdot \vec{\sigma}_{t} / p_{\bar{l}}\right)_{t, \bar{t}}\left(p_{b} \cdot p_{\nu}\right)$.

The correlation density matrix for unpolarized gluon fusion into $t-\bar{t}$ has the form of an angular distribution

$$
\begin{aligned}
W\left(\theta, p, p_{\bar{l}}, p_{l}\right)= & \frac{1}{4}-\frac{1}{4}\left\{\left[p^{4} \sin ^{4} \theta+m^{4}\right]\left(\hat{p}_{\bar{l}}\right)_{x}\left(\hat{p}_{l}\right)_{\bar{x}}\right. \\
& +\left[p^{2}\left(p^{2}-2 m^{2}\right) \sin ^{4} \theta-m^{4}\right]\left(\hat{p}_{\bar{l}}\right)_{y}\left(\hat{p}_{l}\right)_{\bar{y}} \\
& +\left[p^{4} \sin ^{4} \theta-2 p^{2}\left(p^{2}-m^{2}\right) \sin ^{2} \theta+m^{2}\left(2 p^{2}-m^{2}\right)\right]\left(\hat{p}_{\bar{l}}\right)_{z}\left(\hat{p}_{l}\right)_{\bar{z}} \\
& \left.+2 m p^{2} \sqrt{p^{2}-m^{2}} \cos \theta \sin ^{3} \theta\left[\left(\hat{p}_{\bar{l}}\right)_{x}\left(\hat{p}_{l}\right)_{\bar{z}}-\left(\hat{p}_{\bar{l}}\right)_{z}\left(\hat{p}_{l}\right)_{\bar{x}}\right]\right\} \\
& \times\left\{p^{2}\left(2 m^{2}-p^{2}\right) \sin ^{4} \theta+2 p^{2}\left(p^{2}-m^{2}\right) \sin ^{2} \theta\right] \\
& \left.+m^{2}\left(2 p^{2}-m^{2}\right)\right\}^{-1}
\end{aligned}
$$

where $m$ is the top quark mass, $\theta$ is the top quark production angle in the quarkantiquark CM frame, $p$ is the gluon CM momentum, $\hat{p}_{\bar{l}}$ is the $l^{+}$momentum direction in the top rest frame and $\hat{p}_{l}$ is the corresponding $l^{-}$direction in the antitop rest frame. For the polarized gluon case this distribution breaks up into more pieces, depending on the gluons being polarized linearly or not [7].

\section{Summary}

Hyperon polarization is a touchstone for understanding transversity and hence, NPQCD. Regarding the many related phenomena, there are several ways to begin to explain polarization that we have presented. The Fracture Functions and their 
extension into polarization were explored more thoroughly. An outstanding question for experimenters and phenomenologists is how to "see" quark or gluon polarization? We have suggested several approaches to this. In electroproduction we have a measure of the tensor charge. We show, finally, that top quarks provide a nearly direct way to probe quark spin and the SSA sources. Using $t+\bar{t}$ spin correlations could provide a window into linearly polarized gluon distributions at LHC energies.

\section{Acknowledgments}

We thank the organizers of QCD Evolution III 2014 for a productive workshop in a charming setting. This work was funded in part by U.S. DOE grant DE-FG0201ER4120 (S.L.).

\section{References}

1. G. Kane, J. Pumplin and W. Repko, Phys. Rev. Lett. 41,1689 (1978).

2. L. Calero Diaz, P. Di Nezza, G. R. Goldstein, and S. Liuti, in preparation.

3. W. G. D. Dharmaratna and G. R. Goldstein, Phys. Rev. D 41, 1731 (1990); ibid. 53,1073 (1996).

4. T. DeGrand and H. Miettenen, Phys. Rev. D 23, 1227 (1981); ibid 24, 2419 (1981).

5. G. R. Goldstein, Polarization of inclusively produced $\Lambda_{c}$ in a QCD based hybrid model, in Proceedings $\Lambda_{c}$ Workshop, (Fermilab (1999)) and arXiv:hep-ph/9907573 (1999).

6. E. M. Aitala, et al. Phys. Lett. B471, 449 (2000).

7. G. R. Goldstein and S. Liuti, work in progress.

8. G. R. Goldstein and M. J. Moravcsik, Ann. Phys. (N.Y.) 98, 128 (1976); ibid. 142, 219 (1982); 195, 213 (1989).

9. M. Anselmino, et al., Phys. Rev. D 83, 114019 (2011).

10. S. Ahmad, G. R. Goldstein, and S. Liuti, Phys. Rev. D 79, 05014 (2009); G. R. Goldstein, J. O. Gonzalez Hernandez, and S. Liuti, J. Phys. G 39, 115001 (2012).

11. G. R. Goldstein, J. O. G. Hernandez and S. Liuti, arXiv:1311.0483 [hep-ph] (2013).

12. CLAS Collaboration (Bedlinskiy, I. et al.), Phys.Rev.Lett.109, 112001 (2012).

13. G. R. Goldstein, J. O. Gonzalez Hernandez, and S. Liuti, arXiv:hep-ph/1401.0438 (2014).

14. S. V. Goloskokov and P. Kroll, Eur. Pys. J. A47, 112 (2011).

15. L. Trentadue and G. Veneziano, Phys. Lett. B 323, 201 (1994).

16. F. Ceccopieri and L. Trentadue, Phys. Lett. B 660, 43 (2008).

17. M. Anselmino, V. Barone, and A. Kotzinian, Phys. Lett. B 699, 108 (2011)

18. G. R. Goldstein and S. Liuti, Nuovo Cim. C035N2, 327 (2012) and arXiv:1201.0193 [hep-ph] (2012); also: work in progress.

19. D. Sivers, Phys. Rev. D 81, 034029 (2010).

20. G. R. Goldstein, O. G. Hernandez, and S. Liuti, Phys. Rev. D 84, 034007 (2011).

21. S. J. Brodsky, D. S. Hwang and I. Schmidt, Phys. Lett. B 530, 99 (2001).

22. L. P, Gamberg, G. R. Goldstein, and K. Oganessyan, Phys. Rev. D 67, 071504 (2003).

23. A. Bacchetta, F. Conti and M. Radici, Phys. Rev. D 78, 074010 (2008).

24. R. H. Dalitz and G. R. Goldstein, Phys. Lett. B 287, 225 (1992); Phys. Rev. D 45,1531 (1992).

25. Gary R. Goldstein, Spin Correlations in Top Quark Production and the Top Quark Mass, Proceedings of the 12th International Symposium on High Energy Spin Physics, Amsterdam, eds. C. W. deJager et al., (World Scientific, Singapore, 1997), p. 328. 
26. S. Parke and Y. Shadmi, Phys. Lett. B 387, 199 (1996); G. Mahlon and S. Parke, Phys. Rev. D 53, 4886 (1996); Phys. Lett. B 411, 173 (1997); Phys. Rev. D 81, 074024 (2010) .

27. C. Pisano, et al., JHEP 10, 024 (2013). 